E. M. Fonseca Aizpuru, F. J. Nuño Mateo, M. J. Menéndez Calderón, I. M. Arias Miranda, B. Rodríguez Díaz

Servicio de Medicina Interna. Hospital de Cabueñes. Gijón

1. Ooi LL, Leong SS. Splenic abscesses from 1987 to 1995. Am J Surg 1997; 174: 87-93.

2. Chun CH, Raff MJ, Contreras L, Varghese R, Waterman N, Daffner R, Melo JC. Splenic abscess. Medicine 1980; 59: 50-65.

3. Górgolas M, Fernández Giusti A, Azofra J, Ezpeleta C, Barros C, Renedo $\mathrm{G}$, et al. Abscesos esplénicos: Estudio clínico-microbiológico de quince casos. Rev Clin Esp 1991; 189: 278-82.

4. Suárez CG, Bayona JF, Rivas P, Cubero LL, Gorgolas M. Endocarditis and splenic abscesses. An Med Interna (Madrid) 2003; 20: 54-5.

5. Chang KC, Chuah SK, Changchien CS, Tsai TL, Lu SN, Chiu YC et al. Clinical characteristics and prognostic factors of splenic abscess: A review of 67 cases in a single medical center of Taiwan. World J Gastroenterol 2006; 12: 460-4.

6. Ng KK, Lee TY, Wan YL, Tan CF, Lui KW, Cheung YC, et al. Splenic abscess: Diagnosis and management. Hepatogastroenterology 2002; 49: 567-71.

7. Casado L, Hevia C, Colunga, Suárez E, Cartón JA, Arribas JM. Absceso esplénico por Salmonella enteritidis. Drenaje percutáneo con éxito en 2 pacientes. Enferm Infecc Microbiol Clin 1994; 12: 52-3.

8. Kang M, Kalra N, Gulati M, Lal A, Kochhar R, Rajwanshi A. Image guided percutaneous splenic interventions. Eur J Radiol 2007; 64: 140-6.

9. Lucey BC, Boland GW, Maher MM, Hahn PF, Gervais DA, Mueller PR. Percutaneous nonvascular splenic intervention: A 10-year review. AJR Am J Roentgenol 2002; 179: 1591-6.

10. Zerem E, Bergsland J. Ultrasound guided percutaneous treatment for splenic abscesses: The significance in treatment of critically ill patients. World J Gastroenterol 2006; 12: 7341-5.

\section{Endocarditis en electrodo de marcapasos de resincronización cardiaca. Tratamiento quirúrgico con circulación extracorpórea}

\section{Sr. Director:}

La terapia de resincronización cardíaca (TRC) con marcapaso cardiaco (MC) de Resincronización es una modalidad terapéutica establecida en determinados grupos de pacientes con insuficiencia cardiaca.

Presentamos el caso de un paciente con endocarditis tardía y de repercusión, localizada en un electrodo del sistema de MC de resincronización, tratado quirúrgicamente con éxito.

Paciente varón de 60 años, portador de un MC tricameral para resincronizacion (Guidant Contact Renewal TR) desde el 19 de abril del 2005, implantado por bloqueo trifascicular, cardiomiopatía dilatada y disfunción ventricular izquierda con fracción de eyección del $24 \%$.

El 9 de agosto de 2006 ingresó por fiebre vespertina de 4 semanas de evolución. Antecedentes personales de obesidad, diabetes mellitus no insulinodependiente y disfunción eréctil, por lo que se sometió a cirugía de implantación de prótesis de pene en junio de 2006. Tras su ingreso se realizaron hemocultivos siendo positivos para Staphilococcus epidermidis coagulasa negativo, instaurándose tratamiento antibiótico intravenoso (i.v.). Se practicó un ecocardiograma transtorácico que mostraba imagen compatible con verruga en electrodo de MC. Seguidamente se efectuó un ecocardiograma transesofágico (ETE) que demostraba una verruga grande de $7 \times 2 \mathrm{~cm}$ en el electrodo ventricular derecho (VD) (Fig. 1). A pesar del tratamiento antibiótico la evolución

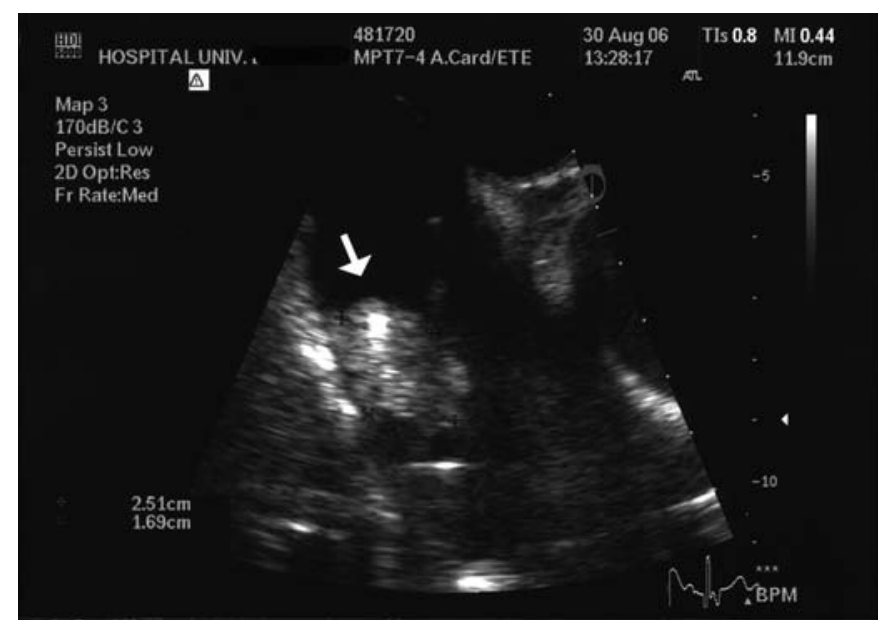

Fig. 1. Ecocardiograma transesofágico preoperatorio. Se aprecia verruga grande en la aurícula derecha en relación con electrodo.

clínica del paciente fue desfavorable, presentando shock séptico, hipotensión arterial, necesidad de inotrópicos, hemofiltración por fracaso renal e ingreso en la Unidad de Cuidados Intensivos (UCI). Ante los hallazgos y la situación clínica se decidió cirugía.

El 7 de octubre de 2006, se intervino quirúrgicamente en situación de urgencia. Se realizó una esternotomía media y bajo circulación extracorpórea (CEC), se retiró el electrodo de electroestimulación del VD con verrugas, electrodo auricular derecho (AD) y electrodo ventricular izquierdo (VI) colocado a través del seno coronario. Además fue extraído el generador situado en región pectoral izquierda. A continuación se implantó en el epicardio de VD, VI y AD tres electrodos epicardicos Medtronic (Medtronic, Inc. USA) que se conectaron a un MC de Resincronización Guidant Contak Renewal TR (Guidant Corporation. Boston Scientific. USA) que se colocó por debajo de la fascia del recto anterior izquierdo del abdomen. La cirugía fue sin incidentes con un tiempo de CEC de 45 minutos y de isquemia miocárdica de 12 minutos. El curso postoperatorio en UCI y sala de hospitalización transcurrió sin complicaciones, siendo dado de alta hospitalaria tras cuatro semanas de tratamiento antibiótico i.v. Desde entonces permanece asintomático y su MC de resincronización funciona correctamente.

La TRC es una forma de tratamiento recientemente introducida en la práctica cardiológica y aplicada a grupos seleccionados de pacientes con insuficiencia cardiaca severa y disincronía mecánica con trastornos en la conducción intraventricular (1). La mayor parte son enfermos con miocardiopatía dilatada o isquémica, habiéndose obtenido mas beneficio con esta terapia en los primeros (1).

En la última década se han expandido las indicaciones e incrementado el número de implantes de dispositivos cardiacos intracavitarios (DCI) como los MC bicamerales, dispositivos de desfibrilación implantable (DAI) y MC de resincronización simples o asociados a DAI. El aumento en el número de casos de implante se ha seguido con un incremento en el número de infecciones.

La incidencia global de infecciones en pacientes tratados de DCI es variable y oscila del 0,2 al 16\%. Según Del Río y cols (2), el $1 \%$ afecta a los electrodos del equipo.

El mejor método diagnóstico de endocarditis en los electrodos de un DCI es el ETE (3). Si el enfermo portador de un DCI tiene bacteriemia y hemocultivos positivos con ausencia de verrugas en el ETE se trata con antibióticos iv. Si además de hemocultivo positivo presenta verrugas en un electrodo, se recomienda la extracción del mismo. Según Klug y cols. (3), si las verrugas al estudio con ETE son menores de $10 \mathrm{~mm}$ se puede considerar la 
extracción percutánea, si son mayores de $10 \mathrm{~mm}$ se indica extracción quirúrgica bajo CEC.

En el caso aquí presentado, dado el tamaño de las verrugas y además de acuerdo con Del Río y cols. (2), se indicó cirugía con CEC.

En el mismo acto quirúrgico, además de la retirada de todo el DCI infectado, consideramos que debe implantarse un nuevo equipo.

Los pacientes con un MC de resincronización al igual que otros portadores de DCI son susceptibles a procesos infecciosos y endocarditis en los electrodos. En los casos con verrugas o/y vegetaciones grandes en algún electrodo con hemocultivos positivos, se recomienda la extracción quirúrgica.

En una revisión en MEDLINE en los últimos 5 años no hemos encontrado un caso similar.

\section{Abad , A. García ${ }^{1}$, L. Caipe ${ }^{2}$, J. Nóvoa ${ }^{1}$, A. Medina ${ }^{1}$}

Servicios de Cirugía Cardiovascular, ${ }^{1}$ Cardiología $y{ }^{2}$ Cuidados Intensivos. Hospital Universitario Dr. Negrín. Las Palmas de Gran Canaria

1. Díaz Infante E. Selección de pacientes candidatos a resincronización cardiaca. En: Mont L, Hernández Madrid A. Resincronización Cardiaca. Barcelona: Marge Medical Books; 2006. p. 17- 98.

2. Del Río A, Angera J, Miró JM, Mont L, Fowler VG, Azqueta M, et al. Surgical treatment of pacemaker and defibrilator lead endocarditis: The impact of electrode lead extraction on outcome. Chest 2003; 124: 14511459.

3. Klug D, Lacroix D, Savayel L, Goullard L, Grandmougin D, Hennequin JL, et al. Systemic infection related to endocarditis on pacemaker leads: Clinical presentation and management. Circulation 1997; 95: 20982107.

Sobre la adecuación e importancia de las publicaciones científicas durante el periodo de residencia: ¿ética o estética?

\section{Sr. Director:}

Lejos han quedado ya aquellos programas y diseños curriculares universitarios en cuyos objetivos no se contemplaba en absoluto la formación integral del alumno sino casi exclusivamente la académica, relegando aspectos tan importantes como la adquisición de actitudes y habilidades clínicas en pro de la más pura erudición. Lejos... ¿ ¿o no tan lejos?

En la década de los ochenta, con la implantación del nuevo plan de enseñanza en las facultades de Medicina así como la reforma del sistema de residencia se atisbaba un cambio radical: en la formación de pregrado por una parte se reorganizaban las áreas de conocimiento, con la consiguiente reestructuración de departamentos universitarios y por otra se introducía el concepto de práctica clínica; en el postgrado se reconocía la vía MIR (médico interno residente) además de la ya conocida de alumnado interno como dos vías para optar al diploma de médico especialista (1-3). Si bien habría que esperar varios años para consolidar la formación MIR como modelo fundamental de especialización, en el que progresivamente el médico en formación iba adquiriendo conocimientos y responsabilidades $(4,5)$.

En este contexto, comienzan a formarse a mediados de los noventa multitud de especialistas cuyos planes docentes contemplaban tres vertientes bien diferenciadas: asistencial, docente e investigadora, a las que se le otorgaba una importancia también muy distinta: el área asistencial se hallaba hipertrofiada enormemente y las áreas docente e investigadora apenas se fomentaban. Paralelamente a esta realidad, se aumenta el número de horas dedicado en pregrado a la docencia práctica sin la previsión por parte del sistema de aumento de número de plazas de profesorado, fenómeno paradójico si tenemos en cuenta la necesidad de más docentes en las sesiones prácticas que en las teóricas.

En la actualidad asistimos a una pugna entre la administración sanitaria, sindicatos médicos y asociaciones de médicos residentes para modelar un "Estatuto del médico residente" que quizá, al igual que otras reformas anteriores $(1,2)$ sea más teórico que práctico (6). Cabría esperar un enfoque más concreto de las directrices formativas durante el periodo de residencia especialmente en lo que se refiere a la investigación como complemento de la labor asistencial: la investigación debe ser un fin y no simplemente un medio más o menos grosero de "cubrir el expediente". Además en los últimos años tanto desde el punto de vista curricular como desde el punto de vista informal la importancia que se da al número de publicaciones científicas firmadas (que no siempre elaboradas) por cada grupo de trabajo va en aumento (7). Esto ha creado un "efecto llamada" de forma que, tal vez inconscientemente, podríamos pensar que es mejor profesional aquel que más publica. Entre el colectivo médico y especialmente entre los más jóvenes, existe un afán desmedido por figurar como firmantes de trabajos enviados para publicar. Esto conlleva un aumento considerable del tiempo dedicado a tareas de investigación y eso siempre que el ansia de figurar vaya acompañado del deseo propio de investigar, en perjuicio muchas veces de las labores asistenciales que no debemos olvidar conforman la primera y principal obligación de todo médico residente. No obstante e independientemente de eso, se ha generado un gran aumento de publicaciones científicas en los últimos años que en muchas ocasiones adolecen de inconsistencia y rigor científico, por no mencionar los múltiples defectos de forma y estilo (8) y que probablemente la única función que posean sea la de ejercitar el intelecto de aquel que las firma (siempre y cuando, claro está, las haya escrito él) $(9,10)$. Y observamos en reuniones, congresos y otros foros de índole diversa autoplagios y publicaciones paralelas de un mismo grupo de trabajo, eso si, colocados los mismos autores de muy variadas formas. Como última reflexión, y apelando a la ética profesional, sería deseable que lo que se remite para publicar además de ser veraz, sea original y propio de quien lo firma, abogando por una mayor honestidad de los autores, editores y seleccionadores.

\section{J. López Castro}

Servicio de Medicina Interna. Complejo Hospitalario de Ourense. Ourense

1. Ley $24 / 1982$, de 16 de junio, sobre prácticas y enseñanzas sanitarias especializadas (BOE 29 de junio de 1982).

2. Real Decreto $127 / 1984$, de 11 de enero, por el que se regula la formación médica especializada y la obtención del título de Médico Especialista (BOE 31 de Enero 1984).

3. Ley 14/1986, de 25 de abril, General de Sanidad (BOE de 29 de abril de 1986).

4. Orden de 27 de Junio de 1989, por la que se establecen las normas reguladoras de las pruebas selectivas para el acceso a plazas de Formación Sanitaria Especializada (BOE 28 de Junio 1989).

5. Real Decreto 931/1995, de 9 de Junio, por el que se dictan normas en relación con la formación especializada en Medicina Familiar y Comunitaria de los Licenciados en Medicina a partir del 1 de Enero de 1995 y se adoptan determinadas medidas complementarias (BOE 16 de Junio de 1995). 